UCRL-JC-131603

PREPRINT

\title{
The Large Scale U.S. Dark Matter Axion Search
}

\author{
D. Kinion \\ K. van Bibber
}

This paper was prepared for submittal to the Proceedings of the Axion Workshop

Gainesville, FL

March 13-15, 1998

August 1, 1998

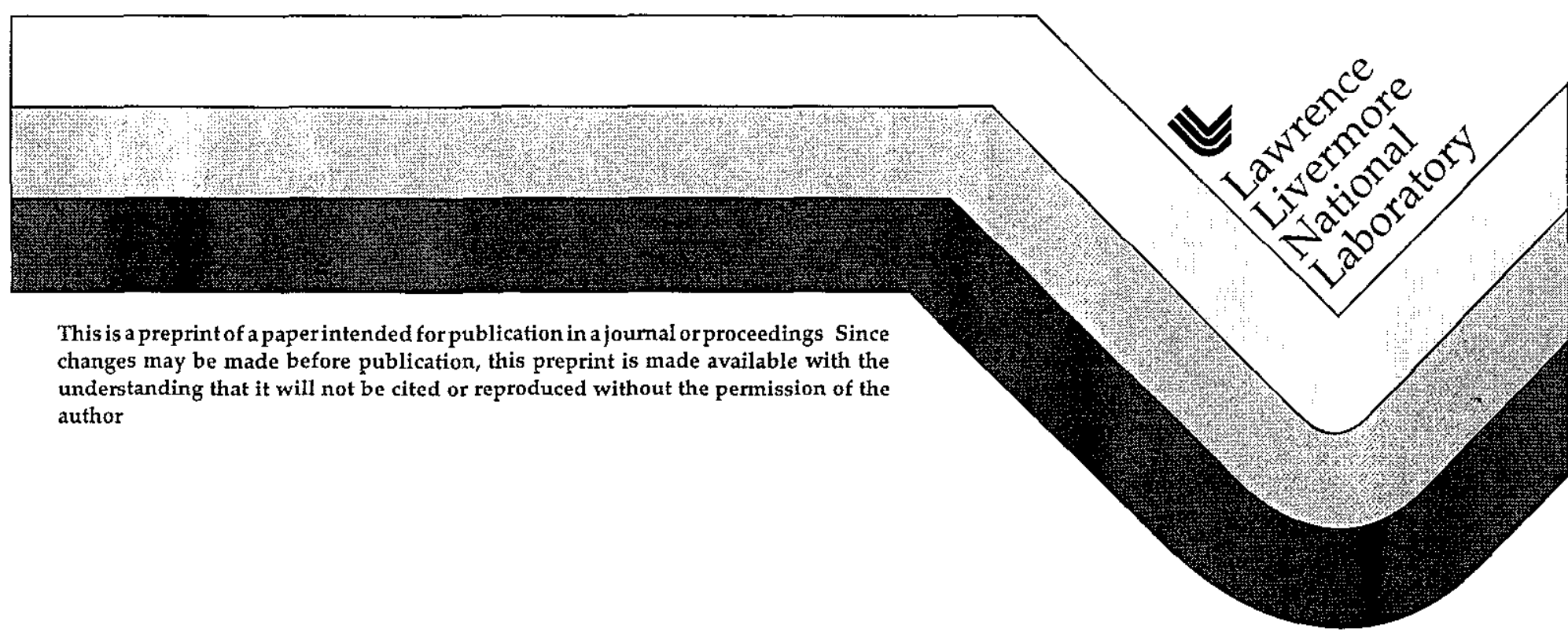




\section{DISCLAINIER}

This dociument was prepared as an account of work sponsored by an agency of the United States Government Neither the United States Gov ernment nor the University of California nor any of their employrees, makes any warranty, express or implied, or assumes any legal liability or responsibility for the accuracy, completeness, or usefulness of any information, apparatus, product, or piocess disclosed, or represents that its use would not infringe pris ately owned rights Reference herein to ant specific commercial product, process, or service by trade name, trademark, manufacturer, or otherwise, does not necessarily constitute or imply its endorsement, reconumendation or favoring by the United States Government or the University of California The views and opinions of authors expressed herein do not necessarily state or reflect those of the United States Government or the Univ ersity of California and shall not be used for adt ertising or product endorsement purposes 


\section{The Large Scale U.S. Dark Matter Axion Search}

D Kinion and $K$ van Bibber $^{2}$

${ }^{a}$ Lawrence Livermore National Laboratory

7000 East Ave, Livermore, CA 94550

We describe the instrumentation and operations of the microwave cavity axion detector presently taking data at Lawrence Livermore National Laboratory This experiment, a collaboration of LLNL, MIT, Univ of Florida, LBNL, Univ of Chicago, FNAL, and INR/Moscow, has been operating with greater than $90 \%$ live time since February 1996 with the objective of exploring the region from 05 to $19 \mathrm{GHz}(21$ to $79 \mu \mathrm{eV})$ at greater than KSVZ sensitivity In a companion paper (E Daw) in these proceedings, the data analysis and first results will be described (See also [1])

\section{INTRODUCTION}

To date, the most efficient method of searching for dark matter axions comprising the halo of our galaxy is the microwave cavity technique originally proposed by Sikivie [2] In a static background magnetic field, axions will decay into single photons via the Primakoff effect The energy of the photons is equal to the rest mass of the axion with a small contribution from its kinetic energy, hence their frequency is given by

$h \nu=m_{a} c^{2}\left(1+O\left(10^{-6}\right)\right)$

At the lower end of the axion window (allowed mass range, presently $1-1000 \mu \mathrm{eV}$ ), the frequency of the photons lies in the microwave regime A high- $Q$ resonant cavity, tuned to the axion mass will enhance the conversion process as well as serve as the detector for the converted photons The expected signal power normalized to typical experimental parameters is $[2,3]$

$$
\begin{aligned}
P_{a \rightarrow \gamma} \approx & 10^{-21} W\left(\frac{B}{77 T}\right)^{2}\left(\frac{V}{200 l}\right)\left(\frac{C}{065}\right) \\
& \left(\frac{Q}{90000}\right)\left(\frac{f}{07 G \tilde{H} z}\right)\left(\frac{\rho_{a}}{\rho_{\text {halo }}}\right)
\end{aligned}
$$

where $\mathrm{B}$ is the background magnetic field, $\mathrm{V}$ is the cavity volume, $\mathrm{C}$ is a mode dependent form factor, $Q$ is the loaded quality factor, $f$ is the resonant frequency, and $\rho_{a}$ is the local halo axion den- sity For the parameters of this experiment, the power from KSVZ axions is typically $5 \times 10^{-22} \mathrm{~W}$

Since the axion mass is unknown, the frequency of the cavity must be tunable For a given signalto-noise ratio (SNR) the scanning rate is

$$
\frac{d \nu}{d t} \approx\left(\frac{25 M H z}{\text { month }}\right)\left(\frac{4}{S N R}\right)^{2}\left(\frac{6 K}{T_{s}}\right)^{2}
$$

where $T_{s}=T_{c}+T_{a}$ is the system noise temperature, specifically the sum of the physical temperature of the cavity $T_{c}$ and the noise temperature of the amplification chain $T_{a}$

Ours is not the first experiment to use this technique to search for axions Two pilot experiments, one at the University of Florida (UF) [4], and another at Brookhaven National Laboratory with collaborators from the University of Rochester and Fermilab (RBF) [5] were carried out in the late 1980s Both experiments fell short of KSVZ sensitivity by 1-2 orders of magnitude Figure 1 shows the exclusion plot of these two experiments along with the goal of the present search, while Table 1 compares the important parameters for all three searches

Although they didn't reach KSVZ sensitivity, the two pilot experiments demonstrated the viability of cavity axion searches A scaling up of parameters $\left(B^{2} V\right)$ and improvements in cryogenic amplifier technology enabled us to scan at KSVZ sensitivity with a reasonable search rate for the first time In this paper, we describe the appara- 
Table 1

Important parameters of the various cavity axion searches Also included are typical values for the proposed upgrade

\begin{tabular}{lrrrr}
\hline & RBF & UF & Present Expt & Upgrade \\
\hline Cavity Volume $(l)$ & $1-11$ & 7 & 200 & 200 \\
B $_{o}(\mathrm{~T})$ & $58-75$ & 75 & 76 & 12 \\
Stored Energy $\left(\mathrm{T}^{2} \mathrm{~m}^{3}\right)$ & $006-031$ & 039 & 110 & 280 \\
Coverage $(\mathrm{GHz})$ & $109-393$ & $132-144$ & $05-19$ & $05-19$ \\
Cavity Temp (K) & 42 & 20 & 15 & $<03$ \\
Cryo Amp Temp (K) & $8-20$ & 30 & $15-45$ & $<03$ \\
\hline
\end{tabular}

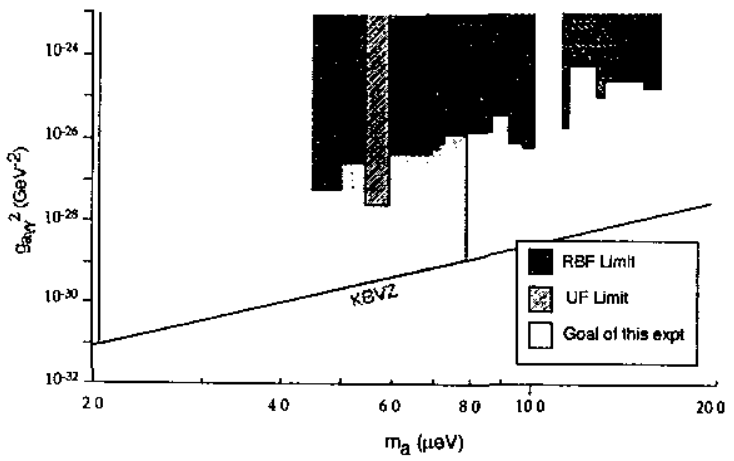

Figure 1 Exclusion plot from the pilot experiments and the goal of this search

tus and operations of this second-generation cavity axion detector

\section{THE SECOND GENERATION CAVITY AXION DETECTOR}

Figure 2 shows the apparatus, consisting of the magnet and the insert containing the cavity and cryogenic amplifiers

\subsection{The Magnet}

The magnet employed in this search is a superconducting NbTi solenoid constructed by Wang NMR [6] It is a low current (224 A), high inductance $(533 \mathrm{H})$ design to maximize field stability, which serves to minimize eddy current heating of the cavity The operating field at the center of the coil is $762 \mathrm{~T}$

For maximum field stability, the magnet should operate in persistent mode, however, the persistent switch failed during construction Instead, the current is supplied by a power supply through a pair of vapor-cooled current leads constructed from copper foil Computer stabilization of the power supply provides field stability of $5 \mathrm{ppm}$, adequate for this experiment, but not for future designs aiming for lower temperatures

The magnet is a warm bore design, meaning that it contains internal thermal shielding between the $42 \mathrm{~K}$ coil and the inner bore This allows the magnet to remain cold whether the insert is in place or not The coil has remained at $42 \mathrm{~K}$ since its arrival in March 1995, using an average of 60 liters of liquid helium per day

\subsection{The Cavity and Tuning Rods}

The microwave cavity is a right-circular cylinder constructed from stainless steel and plated with ultra-high purity, oxygen-free copper Annealing the cavity after plating increased the copper's conductivity The inside diameter is $50 \mathrm{~cm}$ and the length is $1 \mathrm{~m}$

To maximize the form factor from (2)

$C=\frac{1}{B_{o}^{2} V} \frac{\left(\int \bar{E}(x, y, z) \cdot \bar{B}(x, y, z) d V\right)^{2}}{\int \bar{E}^{2}(x, y, z) \epsilon(x, y, z) d V}$

the cavity electric field should be parallel to the static, external magnetic field The $\mathrm{TM}_{010}$ mode has the highest form factor $(C \approx 05-06)$, and is therefore used in the search For this mode, the resonant frequency of the empty cavity is $460 \mathrm{MHz}$, and the unloaded $\mathrm{Q}$ is approximately 200,000

Moving a combination of metal and dielectric rods, running the full length of the cavity changes 


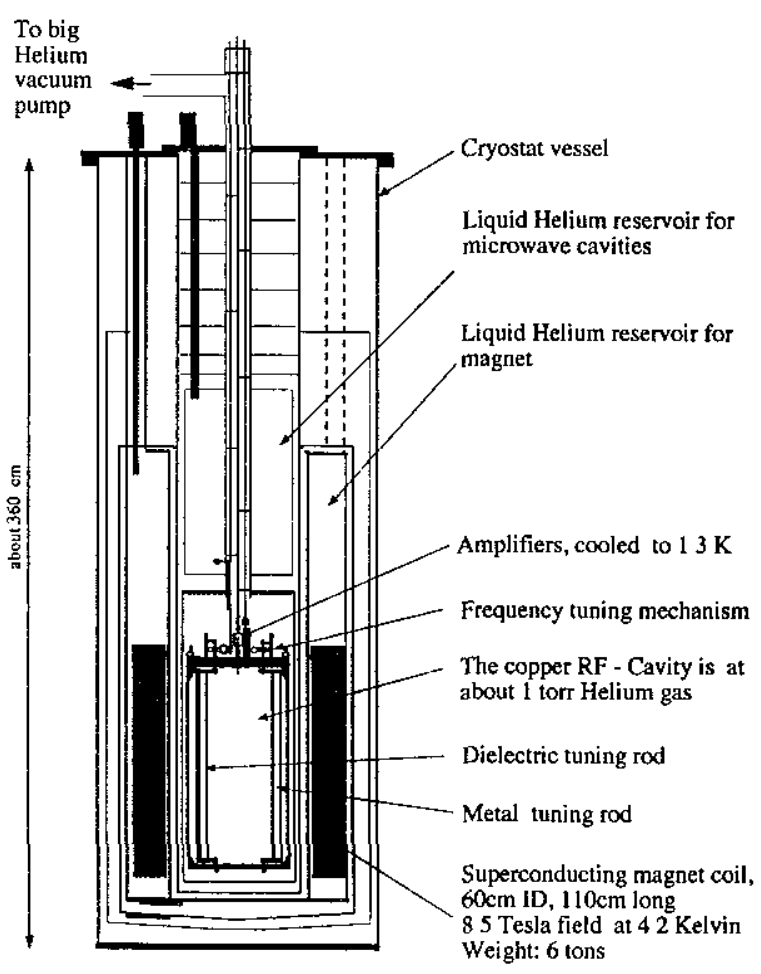

Figure 2 The U S Axion search detector

the resonant frequency These rods can move from the center of the cavity to the wall The single cavity accommodates two rods, Figure 3 shows the frequency coverage allowed by different arrangements

To achieve the required $500 \mathrm{~Hz}$ resolution in resonant frequency it is necessary to move the tuning rods in very fine steps Stepper motors with a resolution of $18^{\circ}$ /step followed by a gear reduction of 300001 control the rods The final step size is approximately $80 \mathrm{~nm}$

An important element of the cavity design is the allowance for the removal of one of the endplates when changing the tuning rod configuration To maintain the high- $Q$ of the cavity, it is important to have a continuous current path between the endplates and the walls A knifeedge seal, held in place by the pressure of ap-

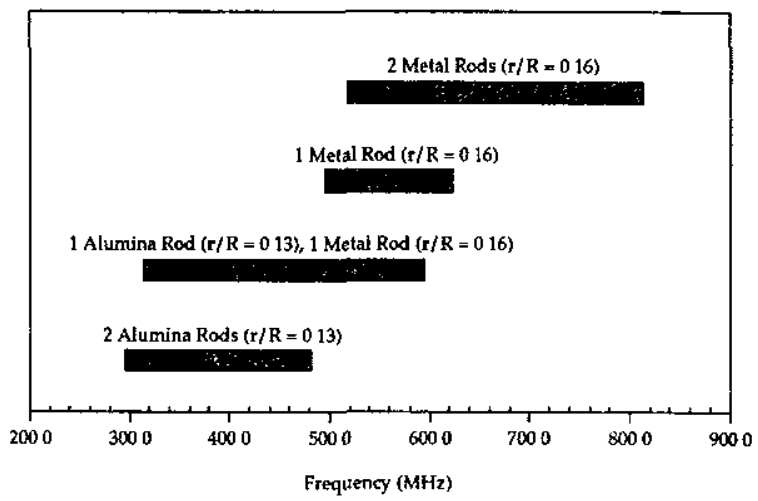

Figure 3 Frequency coverage of different tuning rod configurations in the single cavity

proximately 100 bolts around the circumference, makes this connection We achieve a $\mathrm{Q}$ near the theoretical limit, proving the effectiveness of this type of seal The present cavity has one end fixed and one removable as described above, but the fixed end proves troublesome when plating Because the knife-edge seal works so well, all future cavities will have both ends removable After three years and several openings there has been no noticeable degradation in the $\mathrm{Q}$ of the cavity

\subsection{Insert Cryogenics}

Superfluid ${ }^{4} \mathrm{He}$ maintains the physical temperature of the cavity near $15 \mathrm{~K}$ A capillary tube supplies helium from the 170 liter inner reservoir to a small pool in the bottom of the vacuum can A JT valve in the capillary tube regulates the liquid level A roots blower pumps on the helium, evaporatively cooling the cavity and cryogenic amplifiers to $15 \mathrm{~K}$ The pressure of the helium gas in the cavity is roughly 01 Torr

\subsection{Cryogenic Amplifiers and Cavity Coupling}

The cryogenic amplifiers used in this search are double-balanced GaAs HFET amplifiers supplied by NRAO For details, see the paper by $R$ Bradley The in situ measured noise tempera- 


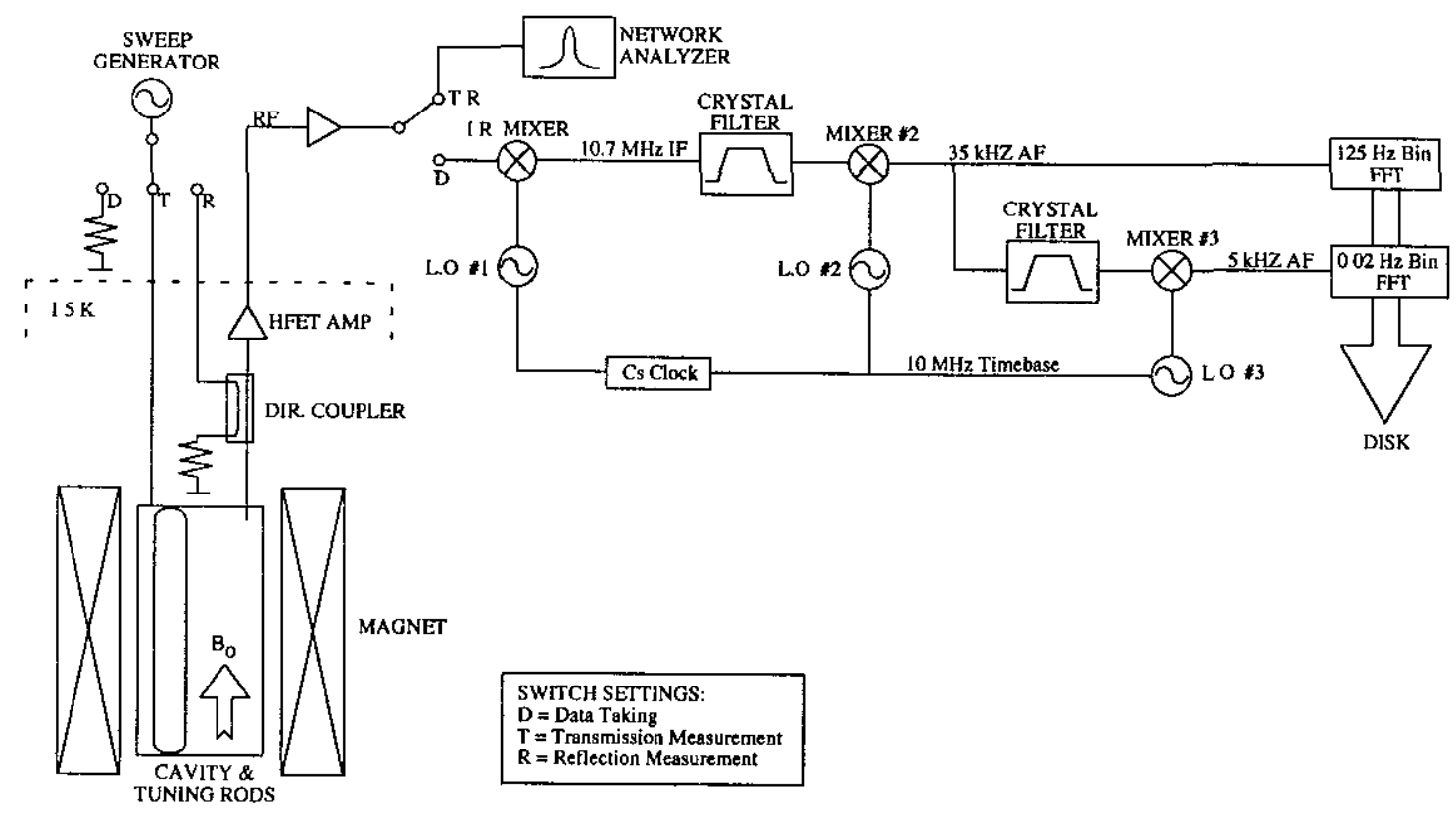

Figure 4 Axion detector schematic

tures range from $17-45 \mathrm{~K}$ For minimum noise temperature, it is important that the amplifiers be positioned so that the $\mathrm{B}$ field is parallel to the plane of the HFET channels [7] Cascading two of these amplifiers achieves sufficient gain $(35 \mathrm{~dB})$ to render downstream noise contributions negligible

The amplifiers are capacitively coupled to the cavity using a short length of low thermal conductivity semi-rigid coax The linear antenna is formed by removing the outer conductor from the last $5 \mathrm{~cm}$ of the coax The strength of the coupling is varied by changing the insertion depth Critical coupling is maintained throughout the run, meaning that on resonance the cavity presents a matched load to the first amplifier

A directional coupler placed between the antenna and the first amplifier allows a direct measurements of the coupling This coupler is used for measurements of the reflection from the cavity The first amplifier is effectively critically coupled to the cavity when this reflection is very small on resonance, typically - $30 \mathrm{~dB}$

A stepper motor similar to the ones used for tuning controls the insertion depth of the an- tenna The resolution is approximately $15 \mu \mathrm{m}$ with a $5 \mathrm{~cm}$ range of motion

\subsection{Room Temperature Electronics}

Figure 4 is a block diagram showing the two major components of the room temperature electronics, the setup for measuring transmission through the cavity and the receiver electronics

Before data is taken at a given frequency, a transmission measurement is made For the measurement, power is fed through a second, very weakly coupled port in the cavity and the transmitted power is measured using a scalar network analyzer A fit of the transmission curve to the sum of a Lorentzian and constant background determines the resonant frequency and $\mathrm{Q}$

The design of the receiver electronics is essentially unchanged from the UF pilot experiment First, the $35 \mathrm{~dB}$ cryogenic amplification described earlier is followed by $35 \mathrm{~dB}$ of roomtemperature post-amplification Next, the signal passes through an image-reject mixer shifting the resonant frequency down to $107 \mathrm{MHz}$ From there, an oven-stabilized, eight-pole crystal filter 
sets the bandwidth of the measurement at $30 \mathrm{kHz}$ This filter also prevents image power from entering the subsequent mixing stages Next, a second mixing stage shifts the center frequency to $35 \mathrm{kHz}$ This audio signal is then sent to both medium and high resolution search channels

The medium resolution search channel consists of a Stanford Research Systems [8] FFT spectrum analyzer The sampling period of the analyzer is $80 \mathrm{msec}$, giving a frequency resolution of $125 \mathrm{~Hz}$ Each step involves averaging 10000 such spectra, resulting in a 400 point power spectrum with 125 $\mathrm{Hz}$ bins These data are coadded and the result searched for Maxwellian peaks a few bins wide (about $700 \mathrm{~Hz}$ ) characteristic of thermalized axions in the halo [9] For a detailed description of the data and its analysis see the paper by $\mathrm{E}$ Daw

An independent, high-resolution search channel operates in parallel to explore the possibility of fine-structure in the axion signal $[10,11]$ The $35 \mathrm{kHz}$ signal passes through a six-pole crystal filter and third mixing stage to shift the center frequency to $5 \mathrm{kHz}$ During the 80 seconds that the medium resolution channel is averaging spectra, a $\mathrm{PC}$ based DSP takes a single 50 second spectrum and performs an FFT The resulting frequency resolution is $20 \mathrm{mHz}$, about the limit imposed by the Doppler shift due to the earth's rotation These data are searched for coincidences between different scans, as well as coincidences with peaks in the medium resolution data

\section{OPERATIONS}

Using LabVIEW [12] software running on a Macintosh computer, this experiment is completely automated, providing 24 hour operation Timbuktu [13], a remote control software package, allows this computer, and consequently the entire experiment to be controlled from any computer with an internet connection This can be across the lab in an office, or across the country

The computer's tasks include regulating the magnet power supply, controlling the LHe level in the vacuum can, tuning the cavity (holding the correct mode), maintaining critical coupling, coordinating the two search channels, and logging the data for analysis In addition, the computer

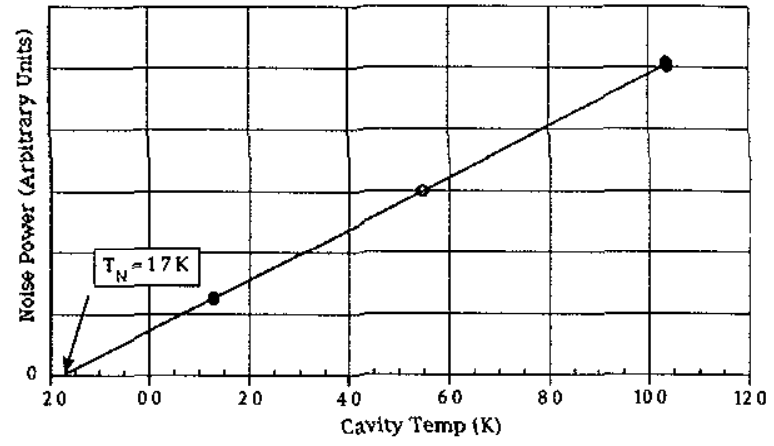

Figure 5 Results from an in situ noise temperature test Several measurements of the noise power were made at three different cavity temperatures

monitors and logs cryogen levels, room and cavity temperatures, magnet coil and lead voltages, pressures, and many other parameters This system has been very reliable, allowing us to run at well over $90 \%$ duty cycle since February 1996

\subsection{Sensitivity Calibration}

The sensitivity of the axion detector is found by measuring the noise power as the physical temperature of the cavity $\mathcal{T}_{c}$ is varied On resonance, the cavity acts like a $50 \Omega$ termination and emits a noise power $P_{c}=k_{B} B T_{c}$, where $k_{B}$ is Boltzmann's constant and $B$ is the bandwidth The noise power at the output of the amplifier is given by $P=k_{B} B G\left(T_{c}+T_{a}\right)$, where $G$ is the gain of the amplifier and $T_{a}$ is its noise temperature

The noise temperature of the amplifier $T_{a}$ is independent of temperature for $T_{c}<12 \mathrm{~K}$, most likely due to inefficient cooling of the HFET channels The temperature dependence of the gain $\mathrm{G}$ can be taken out by following the height of a fixed power peak injected into the cavity during the test The result is a straight-line plot of noise power versus cavity temperature whose intercept gives the noise temperature of the amplifier Figure 5 gives the result from one of these tests 


\subsection{Data taking}

The first scan performed in a region is sequential, with the cavity frequency stepped in roughly $2 \mathrm{kHz}$ intervals The computer adjusts the size of the physical steps to maintain the correct frequency shifts This process is repeated until sufficient integration time is achieved, typically $2-4$ times

This first scan does not always provide uniform coverage, so the software also provides the capability to scan specific regions with differing amounts of integration This provides a flattened signal-to-noise ratio across the region

The final type of scan is the candidate rescan Here, it is necessary to move to a specific frequency, with about $500 \mathrm{~Hz}$ accuracy and perform a long integration The software performs this task, allowing the entry of a text file containing the list of frequencies and the amount of coverage needed For more information about the different scans, see E Daw's paper

The only significant complication with this scheme are mode-crossings, regions where the $\mathrm{TM}_{010}$ frequency is degenerate with either a TE or TEM mode When there are two tuning rods in the cavity, often it is possible to move the locations of these crossings and fill in the gaps in the data When this is not possible, it is necessary to fill the entire cavity with LHe, shifting the frequency of these regions by roughly $3 \%$, before scanning

\section{FUTURE WORK}

There are two major directions for this experiment to go in the future, up in frequency and down in sensitivity Higher frequencies will require smaller cavities, which can be power combined to maintain effective use of the magnet volume Work on this is already underway Going to lower sensitivity, with the ultimate goal of achieving DFSZ, will require a major upgrade

\subsection{Multiple Cavity Arrays}

Exploring higher frequency regions will require increasingly smaller cavities However, placing a single, smaller cavity into the magnet would be inefficient Since the axion signal is coherent over the volume of the cavity $\left(\lambda_{t} \approx 10-100 \mathrm{~m}\right)$, it is possible to power combine the signal from multiple cavities and maintain effective use of the magnet volume

There are several new challenges presented by running with multiple cavities The most fundamental issue is tuning them all sufficiently evenly to maintain balance The mechanical tuning mechanism employed in the single cavity search is not practical when the dimensions of the cavity become small Instead, work is progressing on new, piezoelectric based tuning and coupling mechanisms, providing equal, if not better step resolution

A four-cavity array scheduled for commissioning early in 1999 will be the first realization of a multiple cavity array and piezoelectric tuning With a single copper tuning rod, this setup will cover the region from 13 to $19 \mathrm{GHz}$ Figure 6 shows the preliminary results from a room temperature test of the piezoelectric tuning mechanism, demonstrating the step resolution The step size is controlled by gating a 134 $\mathrm{kHz}$ sinewave For detectable motion, the minimum gate width is $50 \mu \mathrm{sec}$, which when combined with the slope from Figure 6 gives a minimum step size of $550 \mathrm{~Hz}$, well below the necessary 2 $\mathrm{kHz}$ Physically, this corresponds to tuning rod motions on the order of $300 \mathrm{~nm}$

\subsection{Upgrade}

To meet the ultimate goal of scanning at DFSZ sensitivity with sufficient search rate, it will be necessary to perform yet another major upgrade, constituting a third-generation experiment The areas of improvement are the same as for this search over the pilot experiments higher $B^{2} V$ and lower $T$,

Increasing the stored magnetic energy requires the construction of a new magnet Not only should the field be higher (approximately 12T) but it should also be more stable to reduce the eddy current heating in the cavity

There are two ways to lower $T_{s}$, lower the physical temperature of the cavity and lower the noise temperature of the cryogenic amplifiers ${ }^{4} \mathrm{He}$ loses cooling power at $13 \mathrm{~K}$, so going lower in temperature will involve either $\mathrm{a}^{3} \mathrm{He}$ system or a dilution 


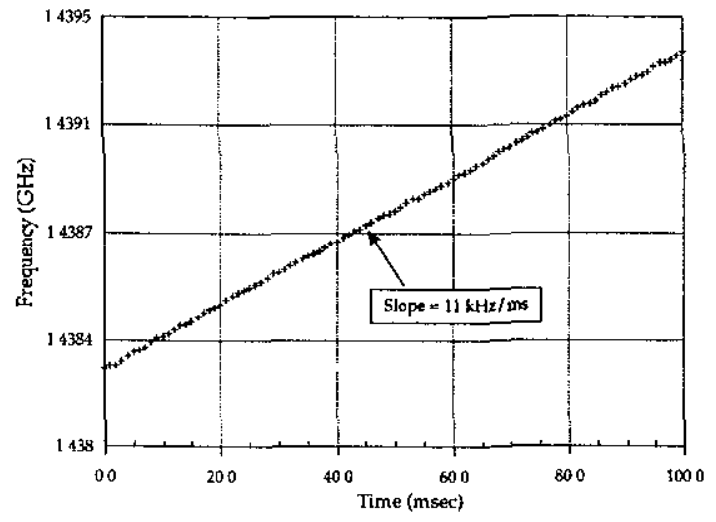

Figure 6 Step resolution of the piezoelectric tuning mechanism For a $50 \mu \mathrm{sec}$ gate width, the step size is $550 \mathrm{~Hz}$

refrigerator Both systems have significantly less cooling power than the current setup, hence the importance of minimizing the eddy current heating Another advantage of the piezoelectric based tuning and coupling mechanisms is the removal of the mechanical connection to the outside of the cryostat and the associated heat loss For details on the new magnet and cryogenic options see the paper in these proceedings by $W$ Stoeffl

New SQUID based RF amplifiers are promising to lower the amplifier noise temperature by at least a factor of $4 \mathrm{M}$ Mück describes these devices in his proceedings paper Combining these with a bigger magnet and lower physical temperature will allow us to scan at DFSZ sensitivity much faster than our present experiment scans at the KSVZ level

\section{CONCLUSION}

Just as the success of the pilot experiments showed the way to get to KSVZ, this experiment has demonstrated that DFSZ axions are within reach In a short time, the technology should be in place to allow the definitive axion search in the lowest decade of the axion window

\section{ACKNOWLEDGEMENTS}

This work was performed under the auspices of the US Department of Energy under contracts no W-7405-ENG-48 (LLNL), DE-FC0294ER40818 (MIT), DE-AC03-76SF00098 (LBL), DE-AC02-76CH03000 (FNAL), DE-FG0297ER41029 (UF), and DE-FG02-90ER40560 (U Chicago), and the National Science Foundation grant number PHY-9501959 (UCB)

\section{REFERENCES}

1 C Hagmann et al, Phys Rev Lett 80 (1998) 2043

2 P Sikivie, Phys Rev Lett 51 (1983) 1415

3 L Krauss et al, Phys Rev Lett 55 (1985) 1797

4 C Hagmann et al, Phys Rev D42 (1990) 1297

5 W U Wuensch et al, Phys Rev D40 (1989) 3153

6 Wang NMR Inc, $550 \mathrm{~N}$ Canyons Parkway, Livermore, CA 94550

7 E Daw and R F Bradley, J Appl Phys 82 (1997) 1925

8 Stanford Research Systems, 1290-D Reamwood Ave, Sunnyvale, CA 94089

9 M S Turner, Phys Rev D42 (1990) 3572

$10 \mathrm{P}$ Sikivie and J Ipser, Phys Lett B291 (1992) 288

11 P Sikivie et al, Phys Rev Lett 75 (1995) 2911

12 National Instruments, $11500 \mathrm{~N}$ Mopac Expwy , Austin, TX 78759

13 Farallon, 14285 Midway Rd Suite 100, Dallas, TX 75244 
